\title{
UTILISATION DE LA TEMPÉRATURE ET DE LA PHOTOPÉRIODE POUR CONTROLER LA MATURATION SEXUELLE EN CAPTIVITÉ DE TROIS ESPĖCES DE POISSONS CYPRINIDÉS EUROPÉENS : BARBUS BARBUS (L.), LEUCISCUS CEPHALUS (L.) ET TINCA TINCA (L.) - RÉSULTATS PRÉLIMINAIRES
}

\author{
P. PONCIN*, Ch. MELARD et J.-C. PHILIPPART**
}

Université de Liège, Service d'Ethologie animale et Aquarium. Laboratoire de démographie des poissons et de pisciculture expérimentale. 22, quai Van Beneden, B-4020, LIĖGE, Belgique.

* Boursier I.R.S.I.A.

** Chercheur qualifié au F.N.R.S.

\section{RÉSUMÉ}

Cet article synthétise les résultats préliminaires de recherches sur le contrôle de la reproduction de barbeaux fluviatiles (Barbus barbus), de chevaines (Leuciscus cephalus) et de tanches (Tinca tinca) élevés dans des conditions comparables de captivité (bassins en circuit semi-fermé ou ouvert, alimentation à satiété), en eau réchauffée et en photopériode naturelle ou manipulée.

L'ovulation est spontanée chez le barbeau et parfois chez la tanche mais elle doit obligatoirement être induite (hypophysation) chez le chevaine ainsi que chez la plupart des tanches.

En photopériode naturelle, la maturité de ces espèces se produit très tôt dans la saison (janvier-février chez le barbeau et le chevaine, avril chez la tanche) par rapport à la reproduction dans la nature (mai-juin chez le barbeau et le chevaine, juin-juillet chez la tanche). Ce phénomène est principalement dû au maintien de températures élevées $\left(20-28^{\circ} \mathrm{C}\right.$ selon les espèces) pendant toute l'année.

Dans ces conditions particulières d'élevage, le nombre de maturations annuelles peut-être multiplié : de 7 à 15 maturités répétées à intervalles d'une quinzaine de jours chez les femelles barbeaux, 2 maturités à 6 mois d'intervalle chez le chevaine.

Il est expérimentalement démontré qu'une décroissance de la durée d'éclairement inhibe la maturité des mâles et des femelles chez les trois espèces. Mais la capacité reproductrice est maintenue en photopériode longue constante $(16,5 \mathrm{~L} / 7,5 \mathrm{~N})$ chez la tanche et le chevaine (sous réserve d'une vérification), longue ou constante courte $(10 \mathrm{~L} / 14 \mathrm{~N})$ chez le barbeau.

Mots-clés : reproduction des poissons,

effet de la photopériode

pisciculture en eau réchauffée.

Barbus barbus, Leuciscus cephalus, Tinca tinca.

\begin{abstract}
USE OF TEMPERATURE AND PHOTOPERIOD IN THE CONTROL OF THE REPRODUCTION OF THREE EUROPEAN CYPRINIDS, BARBUS BARBUS (L.), LEUCISCUS CEPHALUS (L.) AND TINCA TINCA (L.), REARED IN CAPTIVITY. PRELIMINARY RESULTS
\end{abstract}

\section{SUMMARY}

This article synthesizes some preliminary results about the control of reproduction of barbels (Barbus barbus), chubs (Leuciscus cephalus) and tenches (Tinca tinca) reared in similar conditions of captivity (tank, feeding to satiety), in heated water and under a natural or controlled photoperiod.

All female barbels (and some of the tench) ovulate spontaneously, but ovulation in the chub and in most tenches has to be induced by hypophysation. 
Under a natural photoperiod, these three species mature early in the year (January-February in the barbel and chub as shown by figures 2, 3 and 4 ; April in the tench), compared with the natural spawning time (May-June in the barbel and chub; June-July in the tench). This phenomenon is likely to be due to the high temperature $\left(20-28{ }^{\circ} \mathrm{C}\right.$ depending on the species) maintained all year round.

Under these particular rearing conditions the number of annual sexual maturations can be multiplied: 7-15 successive spawnings at 15 days intervals in the barbel (figure 1), 2 spawnings at 6 months intervals in the chub (figure 4).

The experiments carried out demonstrate that an artificial daylength decrease inhibits the maturity of the males and females in the three species (figures 2,3,5 and 6). But the ability to reproduce remains under a long constant photoperiod $(16,5 \mathrm{~L} / 7,5 \mathrm{D})$ in the chub (this has to be experimented further) and the tench (figures 5 and 6$)$, under long or short and constant (1OL/14D) photoperiods in the barbel (figures 2 and 3).

\author{
Key-words : fish reproduction \\ photoperiod effect \\ thermal aquaculture \\ Barbus barbus, Leuciscus cephalus, Tinca tinca.
}

\title{
INTRODUCTION
}

L'influence de la température et de la photopériode sur la reproduction des poissons a déjà fait l'objet de nombreuses études débouchant sur des synthèses (DE VLAMING, 1972; BILLARD, 1979; BILLARD, 1980; WOOTON, 1982).

Dans une optique comparative, cet article réunit sous forme concise une série de résultats originaux récemment obtenus dans notre laboratoire et relatifs à trois espèces de Cyprinidés des régions tempérée et méditerranéenne d'Europe. Le barbeau fluviatile (Barbus barbus) et le chevaine (Leuciscus cepha/us) avaient, à ce jour, été très peu étudiés pour ce qui concerne le contrôle de leur reproduction dans la nature et en captivité (voir HANCOCK et al., 1976, pour Barbus barbus): Ia tanche (Tinca tinca) avait déjà été étudiée en captivité par plusieurs auteurs(BRETON et al., 1980; HOROSZEWICZ, 1981 ; QUILLIER et LABAT, 1981) qui ont suggéré ou démontré un effet de la température et de la photopériode sur la reproduction.

L'étude en laboratoire de l'influence de facteurs environnementaux comme la température et la photopériode aide d'abord à mieux comprendre les mécanismes fondamentaux de la reproduction naturelle des espèces des régions tempérées. Dans la perspective des applications en pisciculture, les études sur le contrôle de la reproduction des poissons visent à élaborer des procédés rendant possible une production plus ou moins continue d'œufs et d'alevins, indépendamment des saisons (BRETON et al, 1983).

Les travaux entrepris par l'Université de Liège à la pisciculture en eau réchauffée de Tihange (centrale électronucléaire sur la Meuse) se situent dans un double contexte de recherche fondamentale (réponse démographique des espèces à la captivité) et appliquée. Ainsi, les expériences relatées dans cet article ont déjà débouché sur une production de plus de 100.000 barbeaux et chevaines utilisés pour le repeuplement des rivières belges et étrangères (PHILIPPART et al., 1984 ; PHILIPPART, 1986 PHILIPPART et al., 1986).

\section{MATERIEL ET MÉTHODES}

Les résultats évoqués dans cet article ont été obtenus en Belgique (latitude : $51^{\circ}$ Nord, longitude : $5^{\circ} \mathrm{Est}$ ). Les installations expérimentales et les contextes étaient différents mais la méthodologie était fondamentalement toujours la même

- élévage des géniteurs en grands bassins $\left(1,5 \mathrm{~m}^{3}\right.$ à $\left.5 \mathrm{~m}^{3}\right)$ placés en circuit ouvert ou semi-fermé et alimenté è eau réchauffée $\left(20-28^{\circ} \mathrm{C}\right.$ selon les espèces) dont la température était maintenue pendant toute l'année au-dessus des valeurs requises pour la reproduction en milieu naturel: $14-16^{\circ} \mathrm{C}$ pour le barbeau (HANCOCK et al., 1976; PHILIPPART, 1982) et le chevaine (PHILIPPART, 1977) et $20-22^{\circ} \mathrm{C}$ pour la tanche (BRETON et al. 1980). Les poissons étaient nourris à satiété au moyen d'aliments (granulés) pour truites ou pour carpes.

- maintien de certains lots en photopériode $100 \%$ naturelle (bassins sous serre) ou naturelle simulée (en bassins occultés) et imposition à d'autres lots de photopériodes manipulées.

- pour deux des trois espèces (barbeaux et: tanches), les lots placés en photopériode naturelle ou manipulée étaient composés à partir d'un même stock originel 2 stocks différents pour les chevaines) adapté depuis sa naissance aux conditions d'élevage en bassin. 


\section{Barbeau}

Les expériences ont été réalisées avec des barbeaux obtenus par insémination artificielle au départ de géniteurs capturés à l'électricité dans deux rivières belges : l'Ourthe (population 1 de 1982) (PHILIPPART, 1982) et la Berwinne (population 2 de 1983). Ces barbeaux ont été élevés en circuit ouvert (bassins de $\left.4 \mathrm{~m}^{2}-1,5 \mathrm{~m}^{3}\right)$ dans les eaux réchauffées $\left(20-25^{\circ} \mathrm{C}\right)$ de la pisciculture expérimentale de Tihange. Pour les expériences décrites dans cet article, le stock expérimental de barbeaux comprend une centaine d'individus (population 1) répartis en 3 lots comparables de 16 femelles et 19 mâles.

Une fois les femelles arrivées à maturité, les expériences de contrôle de la reproduction ont été menées dans un circuit semi-fermé (MELARD et PHILIPPART, 1981), à la même pisciculture de Tihange. La température était maintenue à $20-21^{\circ} \mathrm{C}$ et les bassins étaient spéciălement aménagés pour le contrôle de la photopériode (éclairage artificiel : 2 × $65 \mathrm{~W}$ lampes "true lite").

La ration journalière de nourriture (Trouvit T2 à $45 \%$ de protéines), ajustée en fonction dupoids moyen des poissons (PHILIPPART, non publié), se situait à un niveau suffisant pour couvrir les besoins.

La maturité des femelles était contrôlée par pression abdominale tous les 2-3 jours (tous les mois pour les mâles). Comme la ponte spontanée des femelles n'avait pas lieu, on procédait systématiquement à l'extraction forcée des ovules.

Les résultats présentés dans cet article concernent une saison partielle de reproduction (janvier-mai 1985) en captivité.

\section{Chevaine}

Les chevaines des lots expérimentaux sont nés par insémination artificiellẹ en 1983 (population 1) et 1984 (population 2), à partir de géniteurs sauvages capturés à l'électricité dans l’Ourthe au moment du frai en début juin.

Ils ont été élevés jusqu'à maturité dans les mêmes conditions que les barbeaux mais les expériences de contrôle de la reproduction ont été menées en circuit ouvert à $20-24^{\circ} \mathrm{C}$. Pendant cette phase, la ration journalière distribuée (Trouvit T2) était environ $2 \%$ du poids corporel des poissons.

Dix individus représentatifs de la population 1 furent disséqués tous les 3 mois afin de calculer leur indice gonadosomatique (I.G.S. = poids de la gonade $\times 100 /$ poids total du corps).

L'ovulation des femelles a été induite par injection d'extraits hypophysaires de carpe, selon la méthode décrite pour la carpe (WOYNAROVICH et HORVATH, 1981 ; HORVATH et LUKOWICZ, 1982).

Les résultats présentés concernent des données récoltées d'octobre 1984 à janvier 1986, ce qui couvre une saison de reproduction du chevaine dans la nature et deux "saiśons" en captivité.

\section{Tanche}

Le stock reproducteur de tanches est originaire d'une pisciculture commerciale (S.A. Piscimeuse). Ces tanches ont été élevées en circuit fermé à la pisciculture pilote de Thumaide (température : $\left.25-26^{\circ} \mathrm{C}\right)$.

Les expériences de contrôle de la reproduction par la photopériode ont été réalisées avec des poissons de $200 \mathrm{~g}$ placés dans des bassins de 1,75 $\mathrm{m}^{3}$ spécialement aménagés à cet effet (éclairage: $2 \times 40 W$ dans les bassins occultés).

La ration journalière de nourriture (aliment Trouvit pour carpe $\mathrm{K} 30 / 2$ ) représentait $2 \%$ du poids corporel des poissons.

La méthode d'induction de l'ovulation est la même que celle utilisée pour les chevaines. 1985.

Les résultats présentés concernent des données récoltées pendant la saison de reproduction

\section{RESULTATS}

\section{Barbeau}

Les barbeaux élevés en eau réchauffée atteignent leur première maturité sexuelle à 10-12 mois chez les mâles et 21-22 mois chez les femelles. Ces dernières ont alors une longueur (au creux de la nageoire caudale) minimale de $18 \mathrm{~cm}$ (limites de variation : $18-30 \mathrm{~cm}$ ), ce qui est nettement inférieur à la taille de maturité dans l'Ourthe en Belgique ( $30 \mathrm{~cm}$ minimum à 7 ans; PHILIPPART, 1977).

Dans ces conditions d'élevage, la maturité des femelles est très précoce dansl'année(premières pontes en janvier-février), alors que dans la nature le barbeau ne se reproduit qu'en fin mai-début juin, quand la température atteint $14-16^{\circ} \mathrm{C}$ (PHILIPPART, 1977, 1982). 
En eau réchauffée, les barbeaux femelles expriment en outre un comportement très particulier de maturités répétées (maximum 8 maturités par individu au cours de la première année de reproduction 1984) déjà décrit par PONCIN (1984) et PONCIN et al. (1985). Ce phénomène qui résulte de conditions thermiques, sociales (mâles et femelles regroupés avec un sex ratio de $1 / 1$ ) et nutritionnelles optimales a été observé à nouveau lors de la deuxième année de reproduction (1985) où il a été possible d'obtenir jusqu'à 15 maturités d'une même femelle (fig. 1).

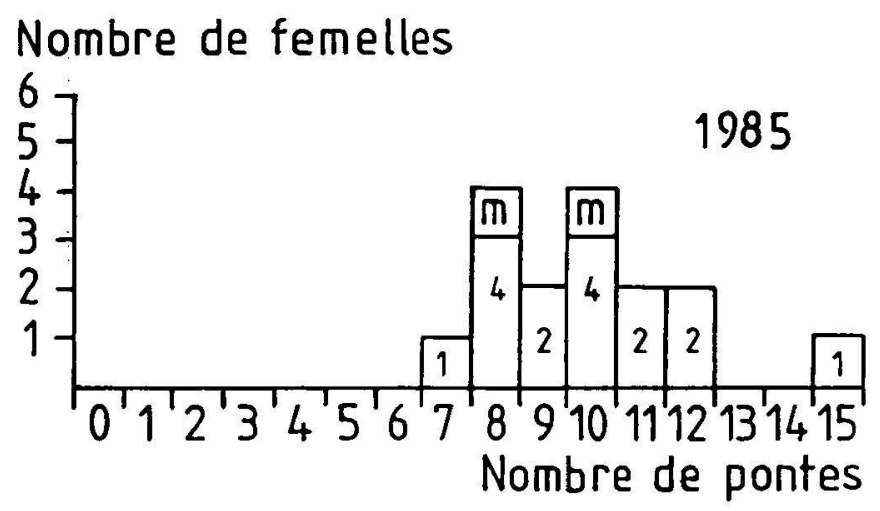

Figure 1 : Nombre de barbeaux femelles ayant pondu de 0 a 15 fois durant la saison de reproduction 1985 (février-août) à la pisciculture de Tihange, en bassin de $1,5 \mathrm{~m}^{3}-4 \mathrm{~m}^{2}$. Lot en photopériode naturelle simulée, à $20^{\circ} \mathrm{C}$. Taille moyenne des femelles (n= 16): $332 \pm 31 \mathrm{~mm}$. Sex ratio en faveur des mâles : 1,2.

"m": femelles mortes en cours d'expérience.

Figure 1 : Number of female barbels having spawned 0 to 15 times during the 1985 (FebruaryAugust) reproductive season, at the Tihange fish research centre, in $1,5 \mathrm{~m}^{3}-4 \mathrm{~m}^{2}$ tanks. Experiment under a natural simulated photoperiod, at $20^{\circ} \mathrm{C}$.

Average length of females (n $=16$ ): $332 \pm 31 \mathrm{~mm}$. Sex ratio: 1,2 male/ 1,0 female.

"m" : dead females.

Abscissa : number of "spawnings".

Ordinate: number of females having spawned.

Même dans ces conditions, la période de maturité des barbeaux captifs ne s'étale toutefois que de janvier à août, moment où la photopériode naturelle, alors décroissante, interrompt le phénomène. Le rôle joué par ce facteur est mis en évidence sans équivoque par une série d'expériences réalisées en 1985 (PONCIN, en préparation). A titre d'exemple, la figure 2 illustre une expérience clé où la décroissance artificielle (hors saison) de la durée d'éclairement inhibe la maturité des femelles.

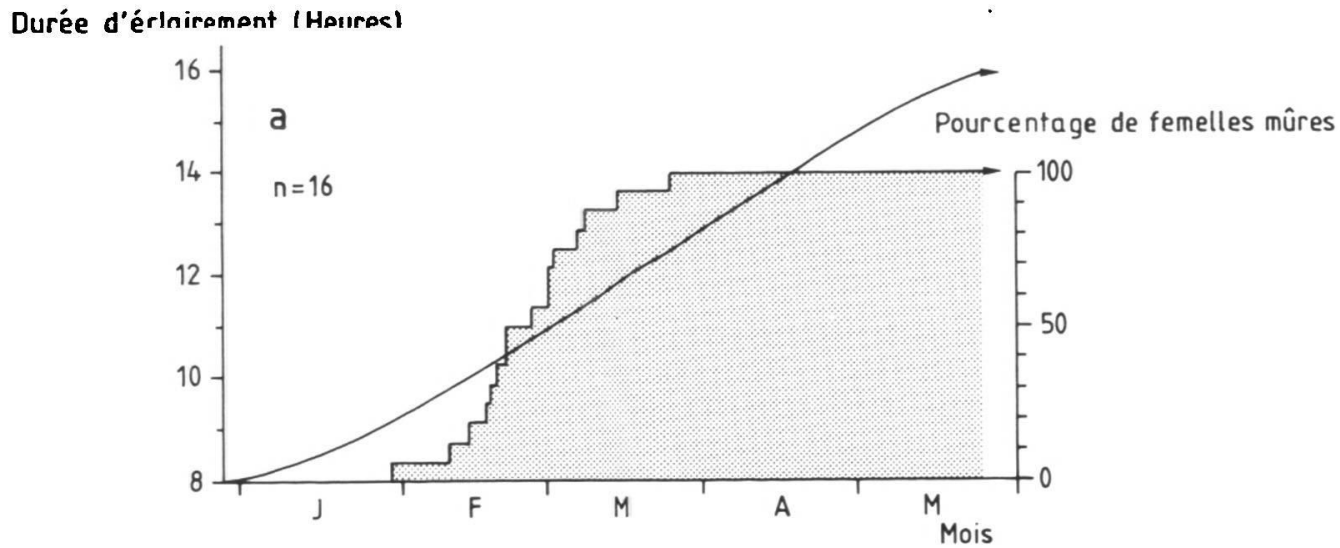


Durée d'éclairement (Heures)

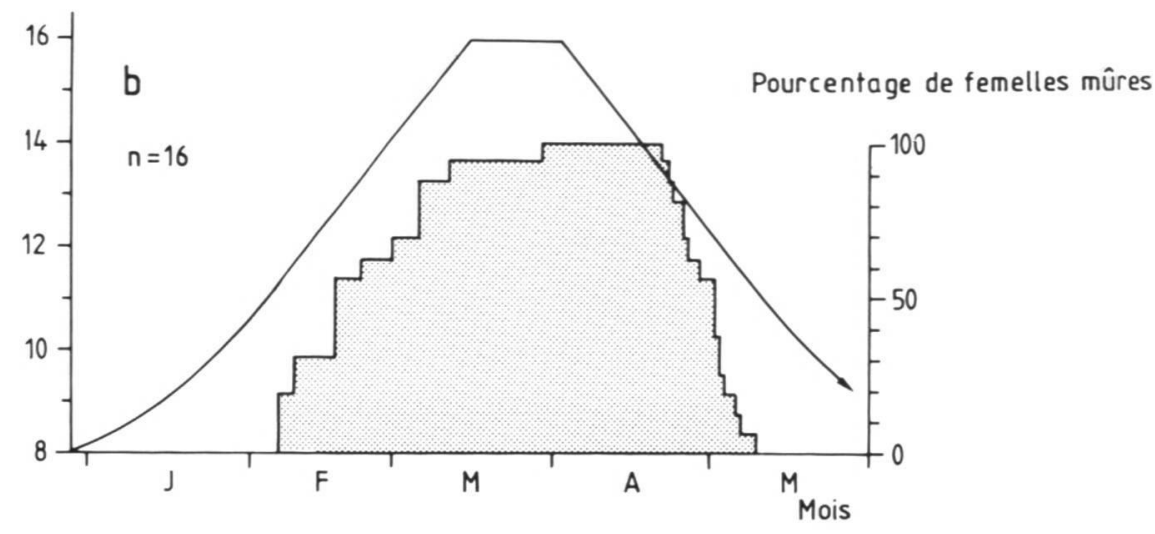

Durée d'éclairement (Heures)

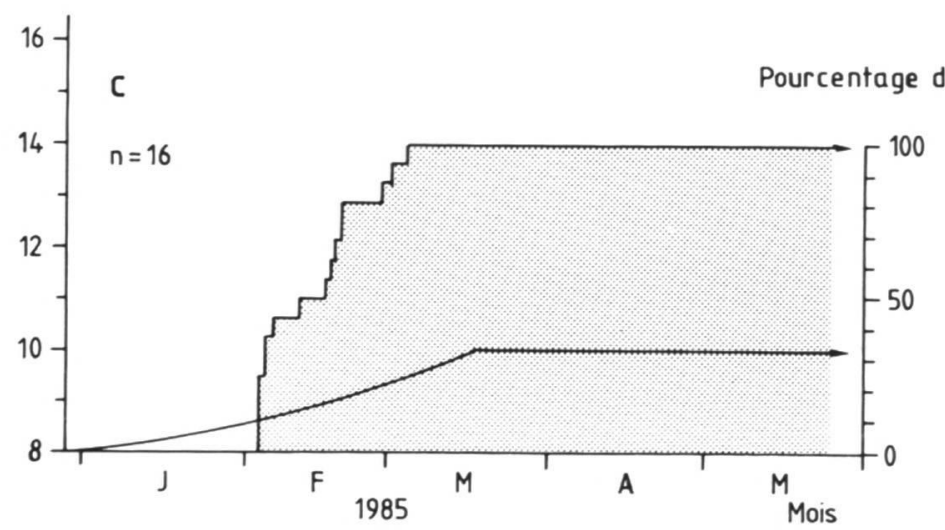

Figure 2 : Pourcentage de barbeaux femelles mûrs dans trois conditions d'éclairement artificiel.

a: cycle naturel simulé

b: cycle annuel condensé en 6 mois

c: photopériode constante au-delà de $10 \mathrm{~L} / 14 \mathrm{~N}$.

L'expérience est menée en circuit semi-fermé avec des femelles (n = 16 par lot) nées en 1982 (population 1) et d'une taille moyenne de 330-335 mm au début des expériences (1985). Sex ratio en faveur des mâles : 1,2.

Figure 2 : Percentage of mature female barbels under three artificial light conditions.

a : simulated natural annual cycle

b: condensed annual cycle

c: constant photoperiod beyond $10 \mathrm{~L} / 140$.

The experiment is carried out in a semi-closed recirculating system with females ( $n=16$ for each experiment) born in 1982 (population 1 ) and with an average length of $330-335 \mathrm{~mm}$ at the beginning of the experiments (1985).

Sex ratio: 1,2 male/1,0 female.

Abscissa : months.

Left ordinate: daylength (in hours)

Rigth ordinate: percentage of mature females.

Un phénomène tout à fait comparable s'observe chez les mâles (figure 3); l'inhibition des mâles du lot b intervient au mois de mai (comme pour les femelles) alors que ceux des lots a et $c$ restent tous spermiants. La seule différence avec les femelles est que les premiers mâles mûrs sont observés en début janvier dans les trois lots, soit un mois avant les premières femelles. 
Durée d’éclairement (Heures)
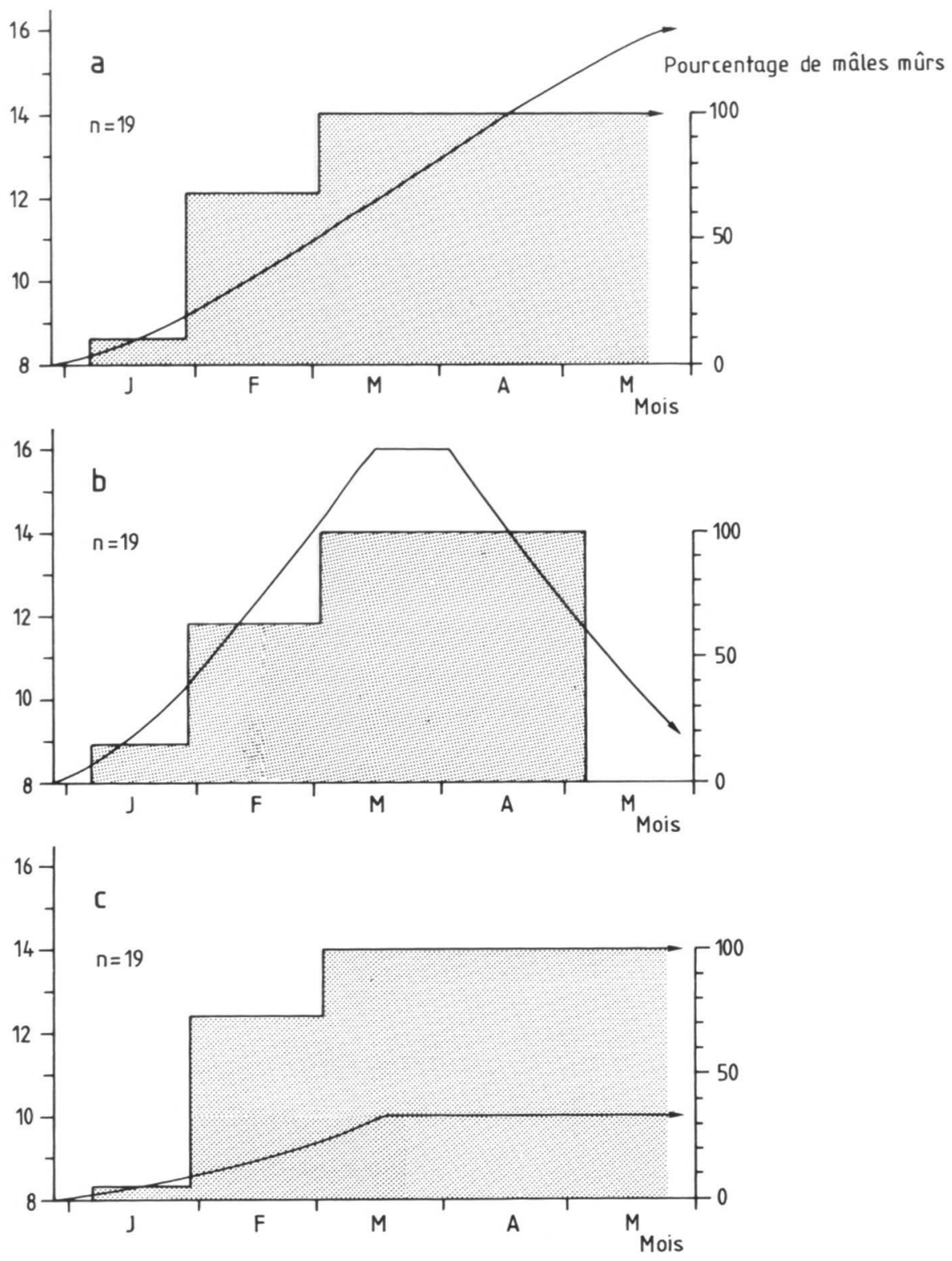

Figure 3 : Pourcentage de barbeaux mâles mûrs dans trois conditions d'éclairement artificiel. a : cycle naturel simulé

b: cycle annuel condensé en 6 mois

c: photopériode constante au-delà de 10L/14N.

L'expérience est menée en circuit semi-fermé avec des mâles (n:= 19 par lot) nés en 1982 et d'une taille moyenne de $205 \mathrm{~mm}$ au début des expériences. Sex ratio : 1.2 mâle/1,0 femelle.

Figure 3 : Percentage of mature male barbels under three artificial light conditions.

a : simulated natural annual cycle

b : condensed annual cycle

c: constant photoperiod beyond $10 \mathrm{~L} / 14 \mathrm{~N}$

The experiment is carried out in a semi-closed recirculating system with males ( $n=19$ for each experiment) born in 1982 and with an average length of $205 \mathrm{~mm}$ at the beginning of the experiments (1985).

Sex ratio: 1.2 male/1,0 female

Abscissa : months

Left ordinate: daylength (in hours)

Right ordinate: percentage of mature males. 
La photopériode joue aussi un rôle modulateur de l'aspect répété des pontes : selon sa văleur, on observe une diminution ou une augmentation du nombre de jours entre deux pontes successives (nombre de jours qui peut passer de 15-20 à 40-50 pour une diminution de 2 heures de lumière). Ce volet des résultats sera traité en détail dans une prochaine publication (PONCIN, en préparation).

\section{Chevaine}

Les chevaines élevés en captivité présentent les premiers signes de maturité à l'âge de 10-12 mois chez les mâles et de 18-26 mois (suivant les conditions d'élevage) chez les femelles. Dans la nature, les mâles comme les femelles atteignent leur première maturité beaucoup plus tard: à l'âge de 2-3 ans pour les premiers et à 4-5 ans pour les secondes (PHILIPPART, 1977).

La maturation des chevaines femelles captifs est spontanée, mais l'ovulation doit être induite artificiellement (hypophysation). Les premières ovulations furent provoquées en juillet $1985: 38,9 \%$ des femelles (sur un total de 18) répondirent en produisant des ovules fécondables en quantités variables (au total : 18.000). Une deuxième hypophysation de ces femelles a été possible 6 mois après la première, c'est-à-dire en janvier 1986, au moment où les mâles arrivaient pour la troisième fois à maturité. Ces résultats sont synthétisés sur la figure 4 qui illustre aussi l'évolution de l'indice gonadosomatique (IGS) des mâles et femelles de 1984 à début 1986 (population1).

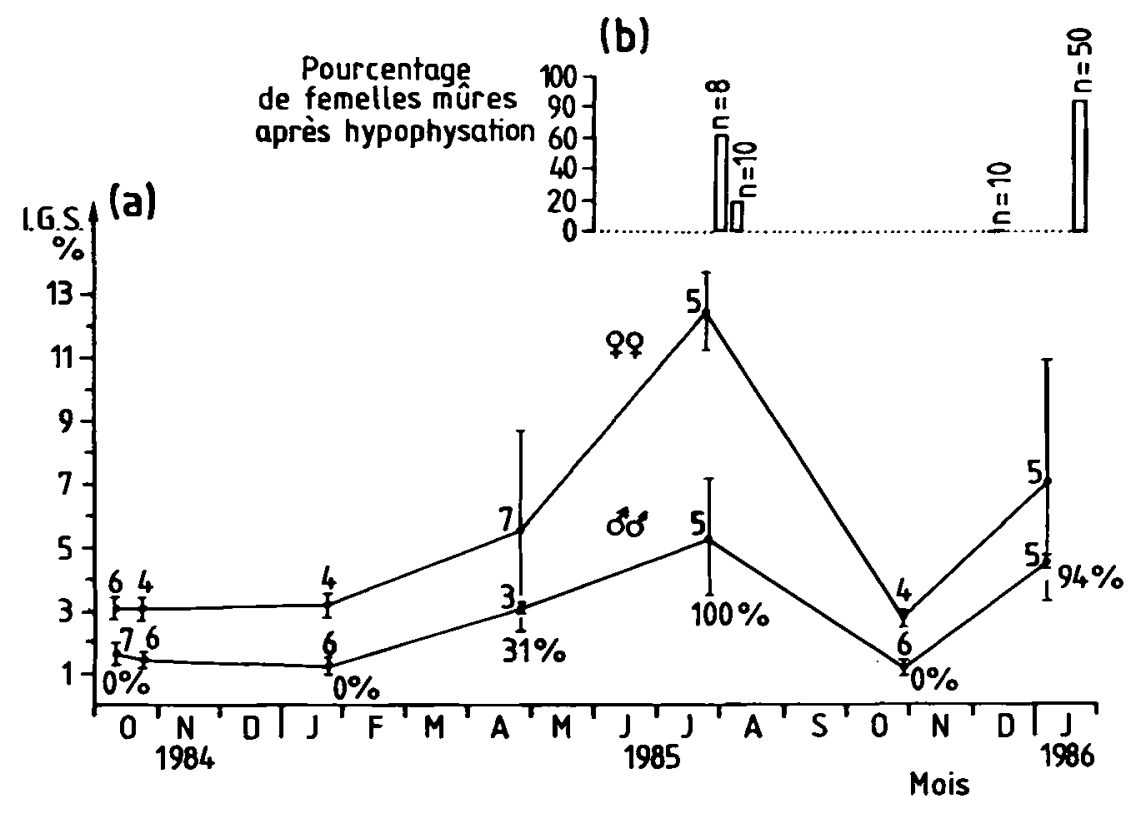

Figure 4 : a) Evolution de I'I.G.S. lindice gonadosomatique: poids de la gonade $\times 100 /$ poids total du corps du poisson) moyen (土 écart-type) chez des chevaines femelles et mâles, d'octobre 1984 à janvier 1986 (température : 20-24 ${ }^{\circ} \mathrm{C}$ ).

Les chiffres indiquent le nombre de poissons disséqués.

Le pourcentage de mâles mûrs est noté pour chaque contrôle.

b) Pourcentage des femelles mûres après hypophysation et nombre total de femelles hypophysées.

Figure 4 : a) Evolution of the average G.S.I. \pm SD (gonadosomatique index $=$ weight of the gonad $\times 100 /$ total body weight) of female and male chubs, from October 1984 to January 1986 (temperature: $20-24^{\circ} \mathrm{C}$ ).

Abscissa : G.S.I.

Ordinate: months

The percentage of mature males is indicated for each control time.

b) ) Percentage of mature females after hypophysation and total number of hypophysated females.

Abscissa : percentage of mature females after hypophysation.

Ordinate: months. 
Comme chez le barbeau, une photopériode décroissante semble inhiber la maturation des chevaines mâles et femelles (figure 5). En effet dans le lot des chevaines maintenus artificiellement en $16,5 \mathrm{~L} / 7,5 \mathrm{~N}$ (population 2) à partir de juin 1985, 66,7\% des femelles répondent favorablement à une hypophysation en décembre 1985 alors qu'aucune réponse ne se produit dans le lot maintenu en photopériode naturelle (population 1). Dans le lot maintenu en 16,5L/7,5N, le pourcentage de mâles spermiants, bien que diminuant de moitié, ne devient jamais nul et se maintient au-dessus des $40 \%$. Ce même pourcentage est nul en octobre dans le lot en basse photopériode naturelle. L'augmentation du pourcentage de mâles spermiants qui apparait dans ce lot à partir d'octobre est le reflet du non-contrôle des poissons à partir de cette période. le contrôle suivant se situant en janvier quand presque tous les mâles avaient à nouveau atteint la maturité.

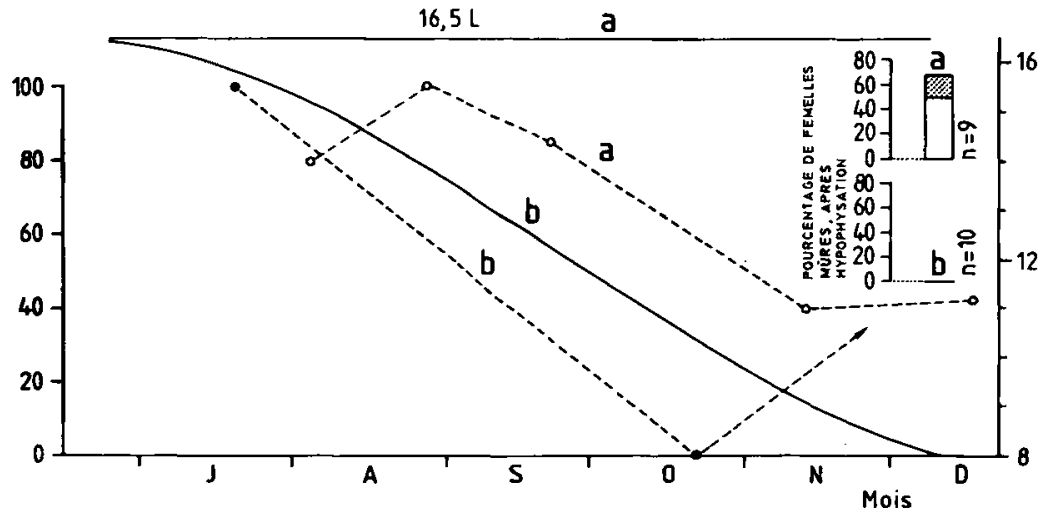

Figure 5 : Pourcentage de mâles mûrs spontanément $(i)$ et de femelles mûres le $11 / 12 / 1985$ (en hachuré : production de quelques ovules seulement) après hypophysation dans deux lots expérimentaux de chevaines âgés de 1,5 ans (population 2 : lot a) et de 2,5 ans (population 1 : lot b) élevés à la pisciculture de Tihange (température $20-24^{\circ} \mathrm{C}$ ).

Lot a: 16,5L/7.5N (/) (superposé l'éclairement naturel: soleil) depuis la fin juin 1985 :

Lot b: photopériode naturelle (/) (soleil).

Les géniteurs mâles et femelles mesuraient de 20 a $28 \mathrm{~cm}$. Lot a: $n=$ 34 mâles; lot $b: n=60$ mâles (juillet) et $n=55$ (octobre).

Figure 5 : Percentage of spontaneously mature males $(i)$ and hypophysated mature (11/12/1985) females (hatching: partial responses, few eggs producted) in two experimental chubs populations (population 2: 1.5 year old, experiment 8 ; population $1: 2,5$ years old, experiment b) reared at the fish research centre of Tihange. Temperature : $20-24^{\circ} \mathrm{C}$.

Experiment a: 16.5/7.5D (/) (superposed to natural daylength) since the end of June 1985 :

Experiment $b$ : natural daylength (/) (sun).

The males and females were $20-28 \mathrm{~cm}$ long. Experiment a: $n=34$ males: Exp. b: $n=60$ males (July) and $n=55$ (October).

\section{Tanche}

Des études très complètes ont démontré l'influence de la température sur la repróduction de la tanche (BRETON et al., 1980; HOROSZEWICZ, 1981). Nos observations apportent quelques compléments à ces résultats.

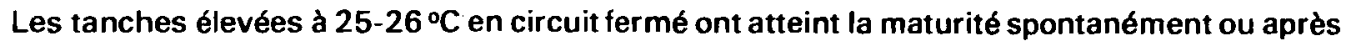
hypophysation, en avril 1985, soit environ 3 mois avant la période de ponte naturelle. 
Pour ce qui concerne l'influence de la photopériode sur la reproduction, nos résultats indiquent que, comme chez le barbeau et le chevaine, une décroissance artificielle de la durée d'éclairement inhibe la maturation des tanches mâles et femelles (fig. 6). Le pourcentage de mâles spermiants diminue parallèlement à la décroissance de la durée d'éclairement tandis qu'il reste constant et élevé lorsque la photopériode est maintenue en $16,5 \mathrm{~L} / 7,5 \mathrm{~N}$. De même, le pourcentage de femelles mûres après hypophysation en novembre est élevé $(46.7 \%$ ) en $16,5 \mathrm{~L} / 7.5 \mathrm{~N}$ (lot a) alors qu'il est nul lorsque la durée d'éclairement diminue (lot b).

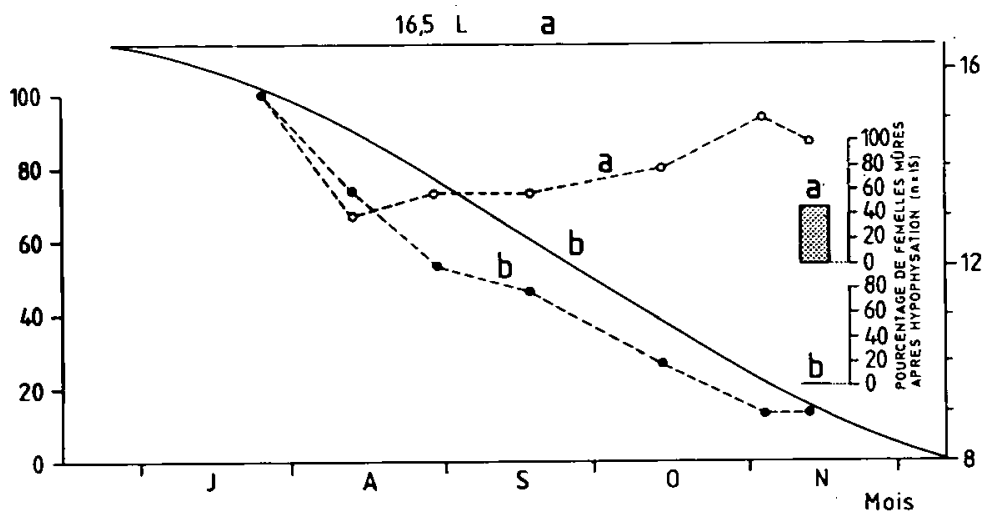

Figure 6 : Pourcentage de mâles $(n=15)$ mûrs spontanément $(i)$ et de femelles (n $=15$ ) mûres le $14 / 11 / 1985$ après hypophysation dans deux lots expérimentaux de tanches élevées à la pisciculture de Thumaide en bassins de $1,75 \mathrm{~m}^{3}$ (température 25-26 ${ }^{\circ} \mathrm{C}$ ).

Lot a : 16,5L/7,5N (/) depuis la fin juin 1985 :

Lot b: éclairement naturel (normal) simulé (/).

Les géniteurs mâles et femelles mesuraient $24,3 \mathrm{~cm}$ en moyenne.

Figure 6 : Percentage of spontaneously mature $(i)$ males (n $=15$ ) and hypophysated mature females $(14 / 11 / 85$ in two experimental tench batches, reared at the Thumaide fish farming centre, in $1.75 \mathrm{~m}^{3}$ tanks (temperature $25-26^{\circ} \mathrm{C}$ ). Experiment a : 16,5L/7,5D (/) since the end of June 1985:

Experiment b : natural simulated daylength (/) (geographical situation of Belgium). The average length of the males and females was $24,3 \mathrm{~cm}$.

\section{DISCUSSION}

\section{Maturité précoce dans la saison}

Chez les trois espèces étudiées, il est possible d'obtenir des maturités précoces : en janvierfévrier chez les barbeaux et les chevaines, en avril chez la tanche. Ce phénomène est principalement dû au maintien pendant toute l'année d'une température élevée qui induit la maturité de ces espèces juste après l'hiver, quand la photopériode faible (9 à 10 heures de lumière en février) mais croissante ne joue aucun rôle inhibiteur (phénomène mis en évidence chez les 3 espèces : voir point 3). Le rôle de certains autres facteurs environnementaux (regroupement des sexes, nourriture à satiété) n'est évidemment pas à négliger mais n'a pas encore été étudié.

Une maturité précoce dans la saison a déjà été observée chez la tanche (BRETON et al., 1980; HOROSZEWICZ, 1981), le gardon (Rutilus rutilus) (MATTHEEUWS et al. , 1981) et la perche (Perca fluviatilis) (PARENT et al., 1976), lorsque ces espèces sont soumises à un réchauffement artificiel de leur milieu (rivière ou étang). 


\section{Multiplication des "pontes" complètes au cours d'une année}

Dans cette partie de la discussion, nous laisserons de côté le cas de la tanche qui, naturellement, pond plusieurs lots d'ovules au cours d'une même saison de reproduction (pontes fractionnées) (HOROSZEWICZ, 1981). Nous discuterons sur les deux phénomènes nouveaux révélés par notre travail:

1) les pontes répétées des barbeaux captifs au cours d'une "saison" de reproduction:

2) les deux reproductions annuelles des chevaines captifs.

Le phénomène des pontes répétées observées chez les barbeaux femelles captifs et, dans une moindre mesure, chez les chevaines, n'est pas propre à ces deux espèces. La carpe peut aussi se reproduire plusieurs fois dans l'année si la température est maintenue à $20^{\circ} \mathrm{C}$ (KOSSMAN, 1975 : cité par BILLARD 1979); GUPTA (1975) montre toutefois, sur la base d'études histologiques, que les carpes femelles élevées en eau réchauffée $\left(23^{\circ} \mathrm{C}\right)$ ne pondent que partiellement. Chez les barbeaux d'élevage, au contraire, chaque ponte successive est complète et correspond à une catégorie bien déterminée d'ovocytes (HANCOCK, 1979).

Des pontes multiples complètes s'observent aussi chez l'épinoche (Gasterosteus aculeatus/qui peut se reproduire jusqu'à dix fois au cours d'une saison de reproduction, lorsque le niveau d'alimentation est suffisant (WOOTON 1977). De cette tactique de reproduction adoptée par les barbeaux femelles d'élevage et par les épinoches se rapproche aussi celle décrite chez le chabot (Cottus gobio) (FOX, 1978; MANN et MILLS, 1979). Dans les rivières calcaires très productives, cette espèce est capable de pondre 4 à 5 fois par saison et la durée de vie moyenne des individus est de 2 à 3 ans ; mais en rivière acide peu productive, le chabot ne pond qu'une seule fois par an et vit en moyenne 10 ans.

La multiplication des pontes d'un poisson au cours d'une saison dépend donc à la fois de la quantité de nourriture disponible et de la température élevée qui permet une plus grande consommation et une meilleure assimilation de la nourriture. Ainsi chez le barbeau, la consommation de larves de Chironomidae vivants est 5 fois plus grande à $20^{\circ} \mathrm{C}$ qu'a $8^{\circ} \mathrm{C}$ (PHILIPPART, communication personnelle). Au vu de ces résultats, on peut se demander quelle sera la longévité des barbeaux d'élevage qui auront produit en 2 à 3 saisons de reproduction autant d'ovules que des barbeaux sauvages pendant toute leur vie.

Chez les chevaines, il semble que la structure ovarienne ne permette que 2 maturités par an (peut-être 3 à l'avenir): Des expériences complémentaires sont en cours pour cọnnaître la capacité maximale de ponte de cette espèce. Pour ce qui concerne les mâles, l'étalement de leur période de maturité correspond à plusieurs "pontes" chez le barbeau mais à une seule chez le chevaine.

\section{Inhibition de la maturité en photopériode décroissante}

Notre étude démontre qu'une durée d'éclairement décroissante (correspondant à des conditions automnales en Belgique) inhibe la maturité des mâles et femelles du barbeau, de la tanche et, sous réserve de confirmation, du chevaine, chez lequel les premiers résultats ont été obtenus avec des poissons issus de deux populations différentes.

La réponse de cés Cyprinidés à une décroissance de la photopériode est tout à fait contraire à celle des Salmonidés chez qui c'est la décroissance de la photopériode qui induit la maturation et la ponte (WHITEHEAD et al., 1978; BRETON et al., 1983; BROMAGE et al., 1984). On comprend ainsi pourquoi, même dans les piscicultures en eau réchauffée, la reproduction des trois espèces étudiées n'a jamais été possible en automne-hiver.

L'influence de phótopériodes courtes sur la reproduction des Cyprinidés avait déjà été évoquée chez le vairon. (Phoxinus laevis) (BULLOUGH, 1941) dont la maturation est retardée par des photopériodes courtes et constantes appliquées après I'hiver et chez la tanche (Tinca tinca) dont la maturation des mâles peut être retardée lorsque ceux-ci sont placés en obscurité totale (QUILLIER et LABAT, 1981). Nos résultats soṇt aussi intéressants à comparer à ceux de HANYU et al. (1982) relatifs à trois espèces de bouvières. Chez deux de ces espèces, Rhodeus ocellatus ocellatus et Acheilognathus tabira, qui se reproduisent au printemps et en été, c'est l'augmentation printanière de la température qui induit la maturation et c'est la diminution post-estivale de la photopériode qui arrête la ponte. En revanche, chez une autre espèce, Pseudoperilampus typus, qui se reproduit en automne, c'est la décroissance de la photópériode qui stimule la maturation et la chute de la température qui arrête la ponte.

Chez les barbeaux, les chevaines et les tanches du milieu naturel, c'est donc très probablement (cela a été démontré pour la tanche: BRETON et al. 1980; HOROSZEWICZ, 1981) l'élévation de la température qui permet la maturation finale et la ponte. la photopériode ne jouant alors qu'un rôle accessoire. Mais c'est là diminution de la photopériode qui empêche la ponte de se poursuivre en août et plus tard, même quand la température est encore favorable. Ce mode de contrôle de la reproduction constitue une adaptation aux conditions environnementales naturelles par laquelle est rendue impossible la production tardive de larves n'ayant, à cause de leur croissance trop faible, presqu'aucune chance de survivre au-delà de l'hiver. 


\section{CONCLUSIONS}

Bien qu'étant encore à un stade préliminaire, cette étude apporte plusieurs résultats nouveaux relatifs au contrôle de la reproduction en captivité chez deux espèces de Cyprinidae d'eau courante, le barbeau et le chevaine, jusqu'ici peu étudiés sous cet angle. Une prochaine publication (PONCIN, en préparation) traitera spécialement de l'influence de la photopériode sur la maturation sexuelle et la reproduction des barbeaux captifs.

En pratique, les techniques combinant le contrôle environnemental et hormonal de la reproduction ont déjà pu être appliquées à la production massive continue d'œufs et d'alevins, surtout chez le barbeau dont la pisciculture de repeuplement est entrée dans une phase de type commercial en Belgique.

Les recherches actuellement en cours visent les objectifs suivants:

- déterminer comment agissent la température et la durée d'éclairement sur la biologie de la reproduction des espèces, les principaux problèmes pris en compte étant les phénomènes de photosensibilité, le rôle des hormones reproductrices ainsi qụe l'influence de l'alimentation.

- appliquer les techniques de contrôle environnemiental de la reproduction à d'autres espèces d'intérêt économique potentiel, notamment le goujon (Gobio gobio) (KESTEMONT, 1985) et diverses espèces de poissons d'ornement.

\section{REMERCIEMENTS}

Les recherches sur le barbeau et le chevaine ont été effectuées au CERER-Tihange (Centre d'Étude pour la Récupération des Énergies Résiduelles) avec l'appui financier de la société productrice d'électricité INTERCOM de PISCIMEUSE S.A., de la Commission Provinciale de Liège du Fond Piscicole et de la Région Wallonne.

Pour l'aide apportée à la réalisation des expériences sur la tanche à la pisciculture de Thumaide (Tournai), nous remercions M. J.C. JAUMOTTE et MIle B. LATOUR de la SIDEHO.

Nous remercions aussi F. ANCIAUX pour sa contribution aux expériences sur le chevaine et $V$. MAAS pour la réalisation des figures.

\section{BIBLIOGRAPHIE}

BILLARD R., 1979. La gamétogenèse, le cycle sexuel et le contrôle de la reproduction chez les poissons téléostéens. Bull. Fr. Pisci., 273, 177-136.

BILLARD R., 1980. Les possibilités de contrôle de la reproduction chez les poissons par modulation des facteurs de l'environnement et manipulations hormonales. Cah. Lab. Montereau, 10 , $11-32$.

BRETON B., HOROSZEWICZ L., BIENIARZK., EPLER P., 1980. Temperature and reproduction in tench: effect of a rise in the annual temperature regime on gonadotropin level, gametogenesis and spawning. II. The female. Reprod. Nutr. Develop, 20 (4A), $1011-1024$

BRETON B., MAISSE G., LEMENN E., 1983. Contrôle phọtopériodique de la saison de reproduction en salmoniculture: une expérience pilote en Bretagne. Bull Fr. Piscic., 288, 35-45.

BROMAGE N.R., ELLIOTT J.A.K., SPRINGATE J.R.C. and WHITEHEAD C., 1984. The effects of constant photoperiods on the timing of spawning in the rainbow trout. Aquaculture, 43, 213-223.

BULLOUGH W. S., 1941. The effect of the reduction of ligth in spring on the breeding season of the minnow (Phoxinus laevis L.). Proceed. Zool. Soc. Lond.. 110, 149-157.

DE VLAMING V.L., 1972. Environmental control of Teleost reproductive cycle : a brief review. J. Fish Biol., 4, 131-140.

FOX P. J., 1978. Preliminary observations on different reproduction strategies in the bullhead/Cottus gobio L.) in northern and southern England. J. Fish. Biol., 12, 5-11.

GUPTA S., 1975. The development of carp gonads in warm water aquaria. J. Fish. Biol., 7, 775-782.

HANCOK R.S., JONES J.W., SHAW R., 1976. A Preliminary report on the spawning behaviour and nature of the sexual selection in the barbel, Barbus barbus (L.). J. Fish. Biol., 9 (1), 21-28.

HANCOK R.S., 1979. The cycle of intraovarian egg size and fecundity of the barbel Barbus barbus in the River Severn. Freshw. Biol., 9, 535-542.

HANYU I., ASAHINA K., SHIMIZU A., 1982. The role of light and temperature in the reproduction cycles of three Bitterlings species; Rhodeus ocellatus ocellatus, Acheilognathus tabira and Pseudoperilampus typus. Proceed. Int. Symp. Reprod. Physiol. Fish, Wageningen, Netherlands, 229. 232. 
HOROSZEWICZ L., 1981. Effect of different thermal regimes on reproductive cycles of tench, Tinca tinca (L.) Part VIII. Towards understanding of reproduction mechanisms and requirements for controlled spawning. Pol. Arch. Hydrobiol., 28, 257-262.

HORVATH L. and LUKOWICZ M., 1982. Tables with data of hatchery procedures and rearing process of some bred warmwater fishes. Aquacultura Hungarica (Szarvas), 3, 212-219.

KESTEMONT P., 1985. Etude des potentialités d'élevage intensif du goujon (Gobio gobio, L.). Comparaison de plusieurs types d'aliments. Bull. Fr. Piscic., 297, 48-54.

KOSSMAN H., 1975. Reproduction experiments on carp (Cyprinus carpio). EIFAC Tech. pap. 25. $122-126$.

MANN R. H. K., MILLS C. A., 1979. Demographic aspects of fish fecundity. Symp. Zool. Soc. Lond., 44, $161-177$.

MATTHEEUWS A., GENIN M., DETOLLENAERE A., MICHA J.C., 1981. Étude de la reproduction du gardon, Rutilus rutilus, et des effets d'une élévation provoquée de la température en Meuse sur cette reproduction. Hydrobiologia, 85, 271-282.

MELARD CH., PHILIPPART J.C., 1981. La production de tilapia de consommation dans les rejets industriels d'eau chaude en Belgique. Cah. Ethol. appliquée, 1/suppl. 2) 120 pp.

PARENT J.P., BOUCHE G. et VALLAS F., 1976. Effet du réchauffement artificiel des eaux sur quelques paramètres physiologiques de deux Téléostéens d'eaux douces: le gardon et la perche. Cah. Lab. Montereau, 3, 5-14.

PHILIPPART J.C., 1977. Contribution à I'hydrobiologie de l'Ourthe. Dynamique des populations et production de quatre espèces de poissons Cyprinidae: Barbus barbus (L.), Leuciscus cephalus (L.). Chondrostoma nasus (L.) et Leuciscus leuciscus (L.). Thèse de doctorat, Université de Liège, $225 \mathrm{p}$.

PHILIPPART, 1982. Mịse au point de l'alevinage contrôlé du barbeau, Barbus barbus (L.), en Belgique. Perspective pour le repeuplement des rivières. Cah. Ethol. appliquée, 2 (2), 173-202.

PHILIPPART J.C., MELARD Ch., PONCIN P., 1984. Réussite de la reproduction artificielle de barbeaux (Barbus barbus (L.)) élevés en captivité. Perspectives pour la mise en place d'un programme de restauration des populations dans le bassin de la Meuse. Cah. Ethol appliquée, 4 (4), 271-278

PHILIPPART J.C., 1986. Programme de restauration des populations du barbeau fluviatile, Barbus barbus (L.) dans le bassin de la Meuse. 1. Genèse du projet et premier résultats obtenus dans la Mehaigne, la Berwinne et la Meuse. Rapport à la Commission provinciale de Liège du Fonds piscicole, $58 \mathrm{pp}$.

PHILIPPART J.C, PONCIN P., MELARD Ch., 1986. La domestication du barbeau fluviatile, Barbus barbus (L.) (Cyprinidae) en vue de la production massive contrôlée d'alevins pour le repeuplement des rivières. Résultats et problèmes. Symposium CECPI/FAO sur la Sélection, l'Hybridation et le Génie génétique Appliqués à l'Aquaculture des Poissons, Mollusques et Crustacés, pour la Consommation et le Repeuplement. Bordeaux, France, 27-30 mai 1986, document E 59.

PONCIN P., 1984. Observations sur le contrôle environnemental de la reproduction du barbeau fluviatile Barbus barbus (L.) en captivité. Effets de la température et de la photopériode Cah. Ethol. appliquée, 4 (4), 357-358.

PONCIN P., PHILIPPART J.C., MELARD Ch., 1985. Induction of repeated spawnings in female barbel, Barbus barbus (L.) (Pisces, Cyprinidae) reared in heated water. 7th conference of the European society for comparative physiology and biochemistry, A 26.

QUILLIER R., LABAT R., 1981. Premières observations sur l'influence de photopériodes constantes sur l'activité teșticulaire de la tanche (Tinca tinca). Bull. Soc. Hist. nat. Toulouse, 116 (1-2), 160-164.

WHITEHEAD C., BROMAGE N.R., FORSTER J.R.M., MATTY A.J., 1978. The effect of alteration in photoperiod on ovarian development and spawning in the trout (Salmo gairdneri). Ann. Biol. anim. Bioch. Biophys., 18 (4), 1035-1043.

WOOTON R. J., 1977. Effect of food limitation during the breeding season on the size, body components and egg production of female sticklebacks (Gasterosteus aculeatus). J. Anim. Ecol., 46. 823-824.

WOOTON R. J., 1982. Environmental factors in fish reproduction. In : RICHTER C.J.J. and GOSS H.J.T. (Editors), Reproductive physiology of fish. Pudoc., Wageningen, 210-219.

WOYNAROVICH E., HORVATH L., 1981. La reproduction artificielle des poissons en eau chaude: manuel de vulgarisation. FAO Doc. Tech. Pêches, (201), 191 p. 